\title{
Downregulation of microRNA-9 increases matrix metalloproteinase-13 expression levels and facilitates osteoarthritis onset
}

\author{
$\mathrm{HONGXIN} \mathrm{ZHANG}^{1 *}, \mathrm{BO} \mathrm{SONG}^{2 *}$ and ZHAOXUN PAN ${ }^{1}$ \\ ${ }^{1}$ Department of Joint Surgery, The No. 89 Hospital of The People's Liberation Army of China, Weifang, Shandong 261021; \\ ${ }^{2}$ Department of Information Engineering, Weifang Vocational College, Weifang, Shandong 261041, P.R. China
}

Received December 9, 2016; Accepted September 28, 2017

DOI: $10.3892 / \mathrm{mmr} .2017 .8340$

\begin{abstract}
Matrix metalloproteinase-13 (MMP-13) degrades collagen and other matrix components, thus playing a critical role in the development of osteoarthritis (OA). The expression level of microRNA-9 (miR-9) is significantly depressed in cartilage tissues of OA patients. Furthermore, bioinformatics analysis demonstrated complementary binding sites between miR-9 and MMP-13. The current study, therefore, investigated whether miR-9 is involved in regulating MMP-13 expression levels and OA onset. Cartilage tissues from OA patients and healthy individuals were compared for miR-9, MMP-13 and collagen type II $\alpha 1$ chain (Col2A1) expression levels. A dual luciferase gene reporter assay was performed to evaluate the association between miR-9 and MMP-13. Sodium iodoacetate was injected into the knee joint cartilage tissues to generate the rat OA model. The expression levels of miR-9, MMP-13 and Col2A1 were compared between the model and control rats. In addition, the OA model rats received miR-9 agomir for further expressional assay. Cartilage tissue samples from the OA patients exhibited significantly lower miR-9 and Col2A1 expression levels when compared with the control rats, whilst the expression level of MMP-13 was upregulated. As the target gene of miR-9, MMP-13 is under the targeted regulation of miR-9. The injection of miR-9 agomir into the knee joint cavity significantly depressed MMP-13 expression in the cartilage tissues of $\mathrm{OA}$ rats, with reduced collagen degradation and enhanced COL2A 1 . OA cartilage tissues have lower miR-9 expression and higher MMP-13 expression levels. Thus, miR-9 inhibits the expression level of MMP-13,
\end{abstract}

Correspondence to: Dr Zhaoxun Pan, Department of Joint Surgery, The No. 89 Hospital of The People's Liberation Army of China, 256 Beigong West Street, Weifang, Shandong 261021, P.R. China

E-mail: zhaoxunpanzxc@163.com

*Contributed equally

Key words: microRNA-9, matrix metalloproteinase-13, osteoarthritis decreases its inhibitory effects on COL2A1, and therefore contributes to antagonizing OA.

\section{Introduction}

Osteoarthritis (OA), also termed degenerative arthritis, aged arthritis or hyperplasia arthritis, is a type of primary/secondary irreversible joint degenerative disorder caused by various factors, and is featured with reactive bone hyperplasia or osteophyte formation at joint ligament attachment sites or subchondral bone $(1,2)$. Therefore, illustration of the OA pathogenesis mechanism from a molecular biology perspective, as well as identification of molecular markers for evaluation of the OA pathogenesis or progression, are critical for OA prevention, drug development, and improving treatment efficacy and prognosis. Matrix metalloproteinases (MMPs) are a proteinase family. It is widely distributed in various mesenchymal tissues, is synthesized and secreted by joint chondrocytes, fibroblasts, synovial cells and neutrophils, and is involved in the degradation of the extracellular matrix (ECM), embryonic development, osteogenesis and cartilage development (3), tumor invasion and metastasis (4). MMP-13 is a member of the collagenase sub-family of MMPs, and degrades type II collagen, which is the featured and abundantly distributed protein in the cartilage matrix, with high specificity (5). MMP-13 upregulation has been demonstrated to be associated with $\mathrm{OA}$ pathogenesis (6). MicroRNA (miR) is a small non-coding mRNA, 20-25 nucleotides in length in eukaryotes, and binds to the 3'-untranslated region (3'-UTR) of target gene mRNA to degrade mRNA or inhibit target gene mRNA translation, thus modulating $>30 \%$ of human gene expression, and participating in the regulation of multiple biological processes, including cell proliferation, differentiation and tissue/organ development (7). Various studies demonstrated significantly decreased miR-9 expression levels in cartilage tissues in $\mathrm{OA}$ patients, indicating its role in $\mathrm{OA}$ pathogenesis $(8,9)$. Bioinformatics analysis identified the complementary binding site between miR-9 and MMP-13. The current study therefore investigated whether miR-9 is involved in regulating MMP-13 expression levels and OA pathogenesis. 


\section{Materials and methods}

Major reagents and materials. Dulbecco's modified Eagle's medium/F12 culture medium, fetal bovine serum, penicillin-streptomycin, and $0.25 \%$ trypsin were purchased from Gibco (Thermo Fisher Scientific, Inc., Waltham, MA, USA). Penicillin-streptomycin was purchased from CellGro (Corning Incorporated, Corning, NY, USA). Type II collagenase was purchased from Sigma-Aldirch (Merck KGaA, Darmstadt, Germany). Lipofectamine 2000 was purchased from Invitrogen (Thermo Fisher Scientific, Inc.). X-tremeGENE siRNA transfection reagent was purchased from Roche Diagnostics (Indianapolis, IN, USA). ReverTra Ace qPCR RT kit (FSQ-101) and SYBR dye were purchased from Toyobo Life Science (Osaka, Japan). micrON ${ }^{\mathrm{TM}}$ agomir-9, micrON ${ }^{\mathrm{TM}}$ agomir-control and miR-9 nucleotide fragment were designed and synthesized by Ribo Life Science Co., Ltd. (Soochow, China). Rabbit anti-MMP-13 antibody (sc-30073), mouse anti-collagen type II $\alpha 1$ chain (COL2A1) antibody (sc-52658) and rabbit anti-COL2A1 (sc-28887) were purchased from Santa Cruz Biotechnology, Inc. (Dallas, TX, USA). Horseradish peroxidase (HRP)-conjugated secondary antibody was purchased from Jackson ImmunoResearch Laboratories, Inc. (West Grove, PA, USA). The dual-luciferase reporter assay system and pGL3-promoter plasmids were purchased from Promega Corporation (Madison, WI, USA).

Experimental animals. Specific pathogen-free grade male Sprague Dawley (SD) rats (age, 8 weeks; body weight, $220 \pm$ 25 grams) were purchased from shanghai SLAC Laboratory Animal Co., Ltd. (Shanghai, China). A total of 30 rats were housed in a specific pathogen-free environment with a temperature of $22 \pm 24^{\circ} \mathrm{C}, 40-70 \%$ humidity under a $12 \mathrm{~h}$ light/dark cycle with free access to food and water.

Clinical information. A total of $24 \mathrm{OA}$ patients who received whole knee joint replacement surgery at the No. 89 Hospital of the People's Liberation Army of China (Weifang, China) between January 2016 and June 2016 were recruited. Tibia samples were collected during surgery. All cases met the diagnostic guideline of OA as stipulated by the American Rheumatology Society (10). OA caused by infection, tumor or rheumatoid disease were excluded. Another cohort of 21 patients, who had undergone post-traumatic amputation, was recruited as a control group from which tibia tissue samples were collected. Informed consents were obtained from all patients, and the study was reviewed and approved by the ethical committee of the No. 89 Hospital of the People's Liberation Army of China (Shandong, China).

Generation and grouping of the OA rat model. Eight-week-old male SD rats were anesthetized with $10 \%$ hydrate chloral via intraperitoneal injection. The skins of the bilateral knee joints were sterilized using $75 \%$ ethanol. Knee joints of the left and right knees were fixed at a $45^{\circ}$ angle. Sodium iodoacetate solution $(4 \% ; 50 \mu \mathrm{l})$ was injected into the right knee joint to prepare the OA model. Focal swelling and motility of the right knee joint indicated successful model generation. Saline $(50 \mu \mathrm{l})$ was injected into the left knee joint. SD rats were sacrificed after 8 weeks of model preparation and joint cartilage tissue samples were collected for further assays.

$\mathrm{SD}$ rats were further randomly divided into 3 groups $(\mathrm{n}=10$ per group). miR-9 agomir $\left(3 \times 10^{3} \mathrm{~mol} / \mathrm{l} ; 20 \mu \mathrm{l}\right)$ was injected into the treated and control knee joint prior to, or 3 weeks after, OA model establishment. The negative control group received an equal volume of agomir control in the left and right knees. A total of $20 \mu \mathrm{l}$ scramble negative control was used in the blank control group at the same time points.

Construction of the luciferase reporter assay gene plasmid. Using HEK 293 genomic DNA as the template, full length fragments of wild type (wt) or mutant (mut) forms of 3'-UTR of the MMP-13 gene were amplified and cloned into pGL-3M plasmid to confirm the association between miR-9 and MMP-13, which was predicted using www.microRNA.org. Recombinant plasmid was subsequently used to transform DH5 $\alpha$ competent cells. Positive clones with correct sequences were screened out by sequencing, and termed pGL3-MMP-13-3'-UTR-wt and pGT-MMP-13-3'-UTR-mut. Luciferase reporter gene assay. Lipofectamine ${ }^{\circledR} 2000$ was used to co-transfect HEK 293 cells (American Type Culture Collection, Manassas, VA, USA) with pGL3-MMP-13-3'-UTR-wt plasmid (or pGL3-MMP-13-3'-UTR-mut) and miR-9 mimic (the wt form of miR-9). Following $48 \mathrm{~h}$ of continuous incubation at $37^{\circ} \mathrm{C}$, a dual-luciferase assay was performed. Briefly, the culture medium was discarded and cells were washed in phosphate-buffered saline (PBS) three times, with the addition of $100 \mu$ l Passive Lysis Buffer. After a 15-min culture, the mixture was centrifuged at $800 \mathrm{x}$ g for $5 \mathrm{~min}$ at $4^{\circ} \mathrm{C}$. The cell lysate $(50 \mu \mathrm{l})$ was mixed with $50 \mu \mathrm{l}$ luciferase substrate and the activity of luciferase was measured immediately. The enzymatic reaction was stopped in $50 \mu 1$ Stop \& Glo (Promega Corporation, Madison, WI, USA), followed by quantification of the sea pansy luciferase activity. The relative expression level of the reporter gene was calculated as the ratio of luciferase activity against sea pansy luciferase activity. The following oligonucleotide sequences were used: 5'-UUCUCCGAACGUGUCACGUUU-3' for scramble negative control and 5'-UCUUUGGUUAUCUAGCUGUA UGA-3' for the miR-9 mimic.

Reverse transcription-quantitative polymerase chain reaction $(R T-q P C R)$. The cartilage tissue samples were homogenized in liquid nitrogen. TRIzol reagent (Thermo Fisher Scientific, Inc.) was added to lyse the cells and RNA was extracted. A ReverTra Ace qPCR RT kit was used to synthesize cDNA from RNA by reverse transcription. Using cDNA as the template, PCR amplification was performed with the addition of SYBR fluorescent dye (Thermo Fisher Scientific, Inc.). PCR conditions were as follows: $95^{\circ} \mathrm{C}$ for $15 \mathrm{sec}$, followed by $60^{\circ} \mathrm{C}$ for $30 \mathrm{sec}$ and $74^{\circ} \mathrm{C}$ for $30 \mathrm{sec}$. Forty cycles were performed on an ABI ViiA TM7 fluorescent PCR cycler. The following primer sequences were used for PCR: Forward, 5'-TCTTTGGTTATC TAGCTGTATGA-3' and reverse, 5'-ACACTCCAGCTGGGT CGCCCTC-3' for miR-9; forward, 5'-ATTGGAACGATA CAGAGAAGATT-3' and reverse, 5'-GGAACGCTTCACGAA TTTG-3' for U6; forward, 5'-TGGACGCCATGAAGGTTT TCT-3' and reverse, 5'-TGGGAGCCAGATTGTCATCTC-3' for COL2A1; forward, 5'-CCAGACTTCACGATGGCA 

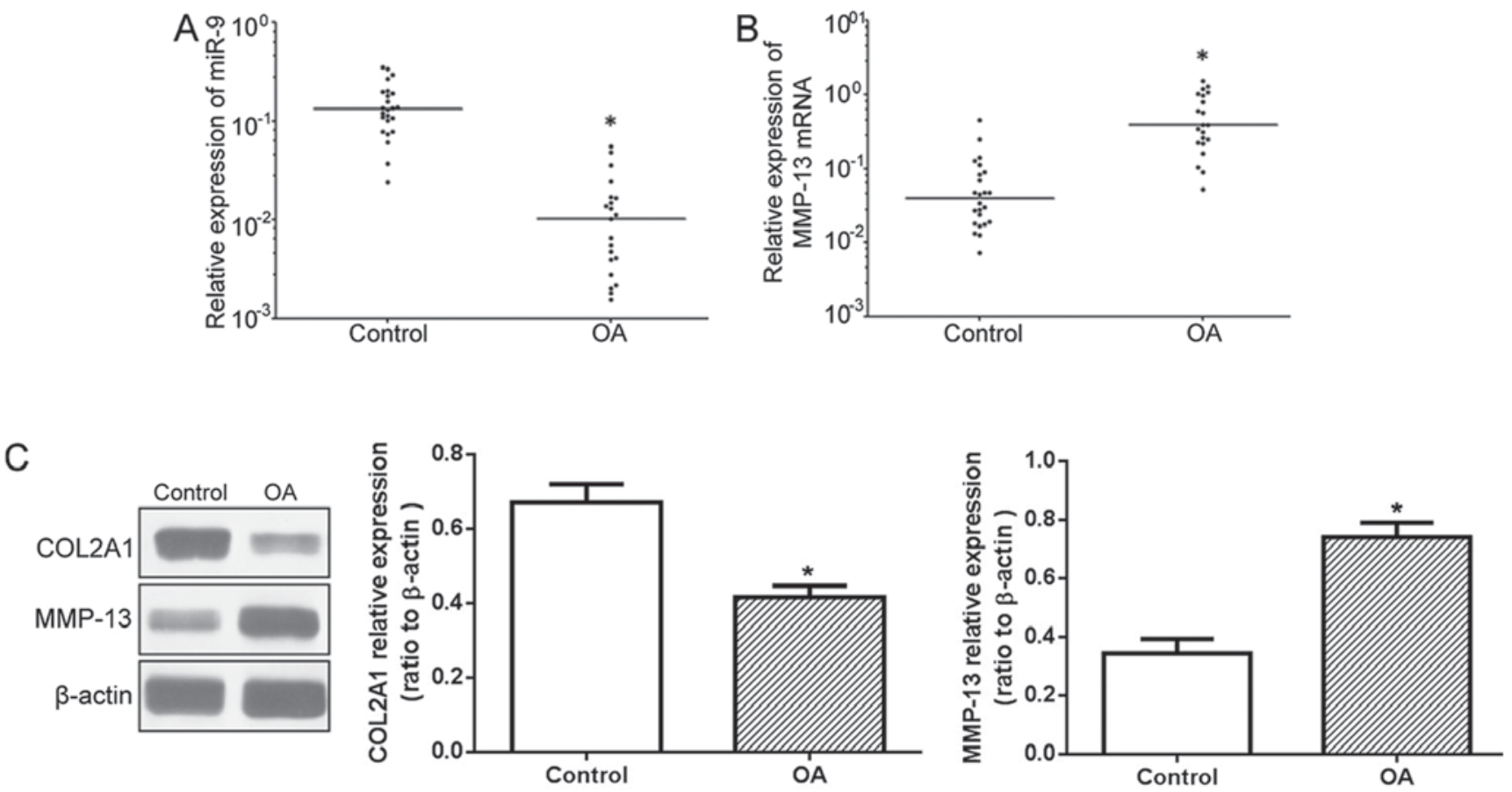

Figure 1. Reduced miR-9 and increased MMP-13 expression levels in cartilage samples from OA patients. Reverse transcription-quantitative polymerase chain reaction for (A) miR-9 expression levels and (B) MMP-13 mRNA expression levels. (C) Western blot analysis of protein expression levels. " $\mathrm{P}<0.05$ vs. control group. miR, microRNA; MMP, matrix metalloproteinase; OA, osteoarthritis; COL2A1, collagen type II $\alpha 1$ chain.

TTG-3' and reverse, 5'-GGCATCTCCTCCATAATTTGGC-3' for MMP-13; and forward, 5'-GAACCCTAAGGCCAAC-3' and reverse, 5'-TGTCACGCACGATTTCC-3' for $\beta$-actin. RNA expression was quantified using the $2^{-\Delta \Delta \mathrm{Cq}}$ method (11).

Western blot analysis. Cartilage tissues were mixed with homogenizing buffer to obtain the tissue lysates. Protein supernatant was prepared after centrifugation at $10,000 \mathrm{x} \mathrm{g}$ for $10 \mathrm{~min}$ at $4^{\circ} \mathrm{C}$. The bicinchoninic acid assay method was used to assess the protein quantity and quality. Protein samples $(80 \mu \mathrm{g})$ were separated in 10\% SDS-PAGE $(3 \mathrm{~h})$ and transferred to polyvinylidene difluoride membrane (wet method, $300 \mathrm{~mA}$ current for $90 \mathrm{~min}$ ). The membrane was blocked in 5\% skimmed milk powder for $60 \mathrm{~min}$, followed by incubation with primary antibodies (anti-MMP-13 at 1:200, anti-COL2A1 at 1:200 or anti- $\beta$-actin at $1: 500$ ) at $4^{\circ} \mathrm{C}$ for $12 \mathrm{~h}$. Following washing (three times) with PBS with Tween 20 (PBST), HRP-labelled secondary antibodies (anti-mouse or anti-rabbit; dilution, 1:8,000) were added and incubated for $1 \mathrm{~h}$ at room temperature. Subsequent to PBST rinsing (three times), the enhanced chemiluminescence reagent was added for a 1-3 min incubation in the dark. The membrane was then exposed in the dark and scanned for data analysis using Quantity One software, version 4.6 (Bio-Rad Laboratories, Hercules, California, USA).

Statistical analysis. SPSS 18.0 software (SPSS, Inc., Chicago, IL, USA) was used for data analysis and the data were presented as the mean \pm standard deviation. Student's t-test was performed to compare measurement data between groups and $\mathrm{P}<0.05$ was considered to indicate a statistically significant difference.

\section{Results}

Reduced levels of miR-9 expression and elevated MMP-13 expression levels in cartilage tissue samples of OA patients. The RT-qPCR results demonstrated significantly reduced miR-9 expression levels in cartilage tissue samples of OA patients when compared with the control group (Fig. 1A), whilst the MMP-13 mRNA expression level was significantly higher (Fig. 1B). Western blot analysis (Fig. 1C) identified higher MMP-13 protein expression levels in OA patients compared with the control group, whereas the COL2A1 protein expression levels were significantly decreased.

Reduced miR-9 and increased MMP-13 expression levels in $O A$ model rats. The RT-qPCR results demonstrated significantly lower miR-9 expression levels in the treated side of the cartilage in the OA model rats, compared with those in the control side, whilst the MMP-13 mRNA expression level was significantly elevated (Fig. 2A). The western blot results demonstrated similar results, as the OA model exhibited higher MMP-13 protein expression levels on the drug treated site compared with the other side, whilst COL2A1 protein expression was downregulated (Fig. 2B).

miR-9 targets and regulates MMP-13 expression. Online prediction identified the targeted binding site between miR-9 and the 3'-UTR of MMP-13 mRNA (Fig. 3A). Transfection of an miR-9 mimic significantly decreased the relative luciferase activity in the HEK 293 cells following transfection with the pGL3-MMP-13-3'-UTR-wt plasmid $(\mathrm{P}<0.05)$, but did not exert a significant effect in the HEK 293 cells transfected with pGL3-MMP-13-3'-UTR-mut 

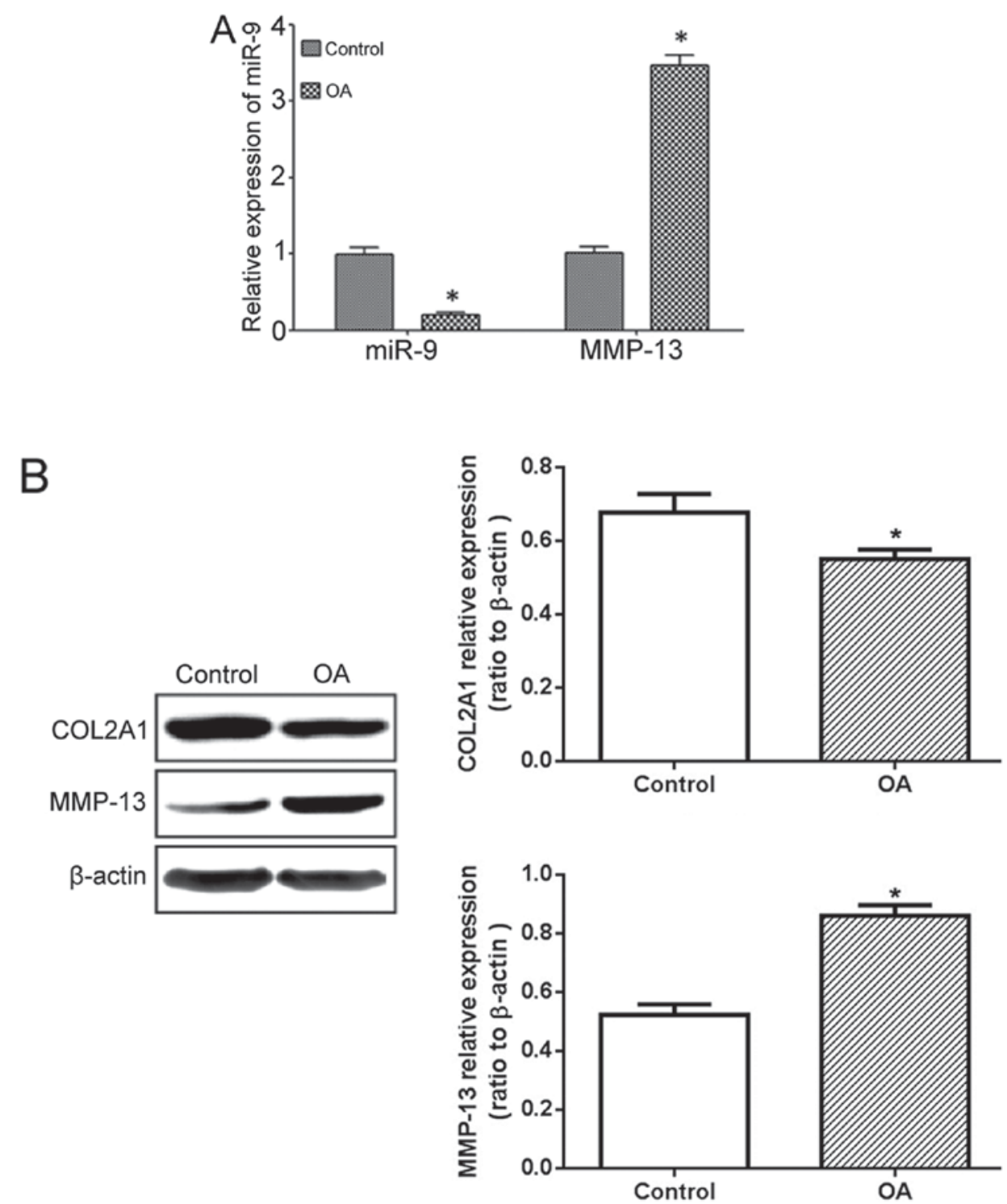

Figure 2. Reduced miR-9 and increased MMP-13 protein expression levels in cartilage tissue samples from OA model rats. (A) Reverse transcription-quantitative polymerase chain reaction for miR-9 expression levels and MMP-13 mRNA expression levels. (B) Western blot analysis of protein expression levels. ${ }^{\text {*P}} \mathrm{P} 0.05$ vs. control group. miR, microRNA; MMP, matrix metalloproteinase; OA, osteoarthritis; COL2A1, collagen type II $\alpha 1$ chain.

\section{AagUAUGUCGAUCUA - UUGGUUUCu 5'hsa-miR-9 || | | | |||||| acAUA - AAAGAAAUAAACCAAAGg 3'MMP13}

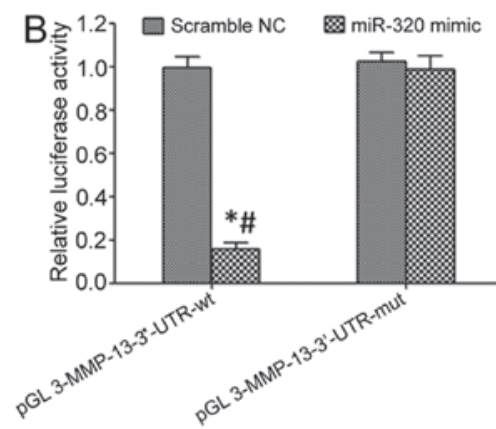

Figure 3. Targeted inhibition of MMP-13 by miR-9. (A) Binding sites between miR-9 and the 3'-UTR of MMP-13 mRNA. (B) Dual luciferase reporter gene assay. ${ }^{*} \mathrm{P}<0.05$ vs. scramble NC group; ${ }^{*} \mathrm{P}<0.05$ vs. pGL3-MMP-13-3'-UTR-mut. MMP, matrix metalloproteinase; miR, microRNA; OA, osteoarthritis; UTR, untranslated region. NC, negative control.
$(\mathrm{P}>0.05)$. These results indicated that miR-9 may target the 3'-UTR of pGL3-MMP-13-3'-UTR-mut and regulate its expression (Fig. 3B).

miR-9 agomir injection decreased MMP-13 expression levels and collagen degradation in cartilage tissue samples of $O A$ model rats. Injection of miR-9 agomir inside the knee joint significantly decreased MMP-13 expression levels in the cartilage tissue samples of OA model rats, with reduced collagen degradation, thus significantly elevating COL2A1 expression levels (Fig. 4A and B).

\section{Discussion}

$\mathrm{OA}$ is the most common type of degenerative disease in human axial and peripheral motor joints. It affects the joint cartilage, subchondral bone, synovial tissues, joint capsule and peripheral muscular tissues, causing a series of clinical symptoms, including joint swelling, pain, aching, stiffness, 
A

$\square$ miR-9 $\square$ MMP-13曰COL2A1

血miR-9 mMMP-13四OL2A1

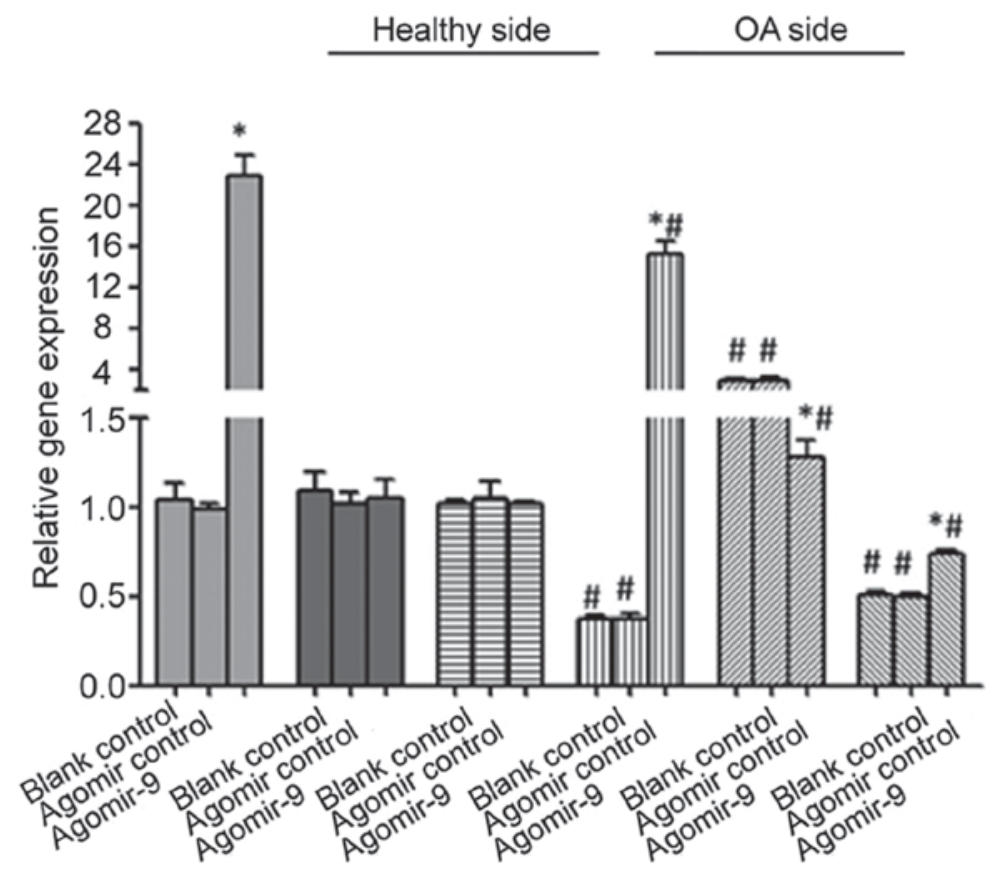

B

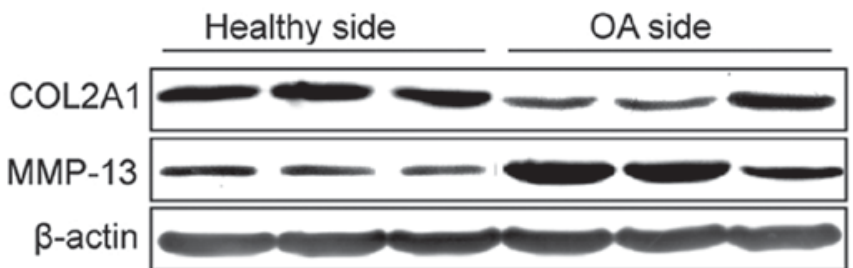

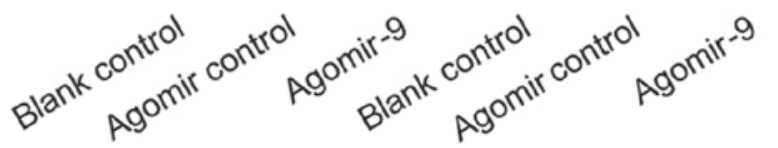
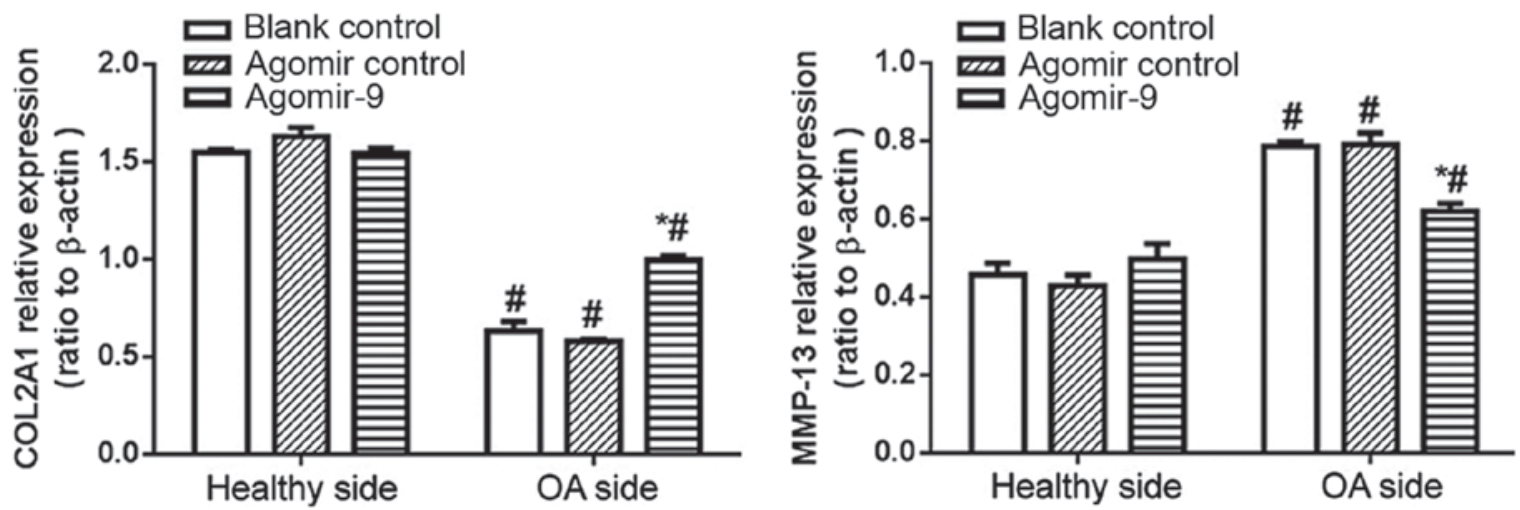

Figure 4. Elevation of miR-9 expression levels decreased the expression level of MMP-13 in cartilage tissue samples from OA model rats and inhibited collagen degradation. (A) Reverse transcription-quantitative polymerase chain reaction for gene expression. (B) Western blot analysis of protein expression levels. ${ }^{*} \mathrm{P}<0.05$ vs. blank control; ${ }^{\#} \mathrm{P}<0.05$ vs. healthy side. miR, microRNA; MMP, matrix metalloproteinase; OA, osteoarthritis; COL2A1, collagen type II $\alpha 1$ chain. OA side received injection of sodium iodoacetate solution and the healthy side (control) received an administration of saline.

atrophy, motility reduction and limitation, thus severely affecting patient quality of life. OA is a degenerative cartilage disease whose incidence increases with age. The number of subchondral feeding vessels rapidly decreases in aged people, causing a loss of elasticity, friction and structural destruction of cartilage (12). The core pathological change of OA is 
impaired joint cartilage plus osteophyte formation and involves multiple factors, including catabolism/anabolism imbalance of cartilage matrix and joint chondrocytes, plus focal inflammation (13-15). Globally, OA has a high incidence; the overall incidence of $\mathrm{OA}$ is $\sim 15 \%$, with a significantly increased incidence in aged individuals from 55 to 74 -years-old (16). OA is prevalent in China, with $>150$ million OA patients, which severely affects the quality of life and working capacity of the aged population (17). The eventual rate of morbidity of $\mathrm{OA}$ is $>50 \%$, making it a major factor for deprivation of working capacity and immobility in aged individuals $(18,19)$. There is currently no highly effective method of reversing OA progression; therefore, the predominant treatment approaches include pain management, deformity correction and recovery or improvement of joint functions (20). Artificial joint replacement surgery is usually required for patients with severe OA. However, the lifespan of artificial joints is only 10 years, therefore it is unfavorable for younger OA patients (21). Thus, further investigation into the pathogenesis of OA is critical for improving treatment efficacy and the quality of life of patients.

Healthy joint cartilage tissues include large regions of ECM, which occupy $99 \%$ of all tissues, leaving just $1 \%$ as joint chondrocytes. Major components of the ECM include water (70-85\%), collagen (10-25\%) and proteoglycan (5-10\%). Type II collagen is the major component of the matrix collagen of cartilage, occupying $80-90 \%$ of total proteins. MMPs are a family of proteinase superfamily and are important in ECM degradation. It is further divided into five groups based on protein structure and reaction substrates, including collagenase (MMP-1, -8 and -13), gelatinase (MMP-2 and -9), tromelysins (MMP-3, -10 and -11), model MMPs (MMP-14, -15, -16, -17, -24 and -25 ) and others (MMP-7, -12, -20 and -23). Collagenase is an important family of MMPs throughout OA pathogenesis, and exerts lysis effects at specific sites. Previous findings demonstrated that abnormally elevated expression levels and activity of collagenase in focal cartilage tissues were important reasons causing the imbalance of catabolism/anabolism of cartilage ECM, and OA pathogenesis (5).MMP-13 is a powerful enzyme with high specificity for degrading type II collagen in the cartilage matrix. Certain studies identified that MMP-13 has approximately 5-10 folds of activity for degrading type II collagen compared with another member of the collagenase family, such as MMP-1 (5). Therefore, MMP-13 performs a major role in degrading the cartilage matrix. Various studies demonstrated significantly enhanced expression levels and activity of MMP-13 in OA cartilage tissue samples (22-25). The degradation of type II collagen (the major component of joint cartilage tissues) by MMP-13 is a major reason causing OA pathogenesis (6). In addition, multiple studies demonstrated significantly reduced miR-9 expression levels in cartilage tissues of OA patients, indicating that miR-9 downregulation is associated with OA onset. Furthermore, bioinformatics analysis demonstrated complementary binding sites between miR-9 and MMP-13. The current study therefore investigated whether miR-9 was involved in regulating MMP-13 expression levels and OA pathogenesis.

Results of the present study demonstrated significantly reduced miR-9 expression levels in cartilage tissue samples from OA patients when compared with the healthy control group, whilst the MMP-13 mRNA expression level was greater.
Western blotting indicated markedly elevated MMP-13 expression levels in OA patient chondrocytes, whilst the COL2A1 protein expression level was significantly lower. In addition, animal experiments showed reduced miR-9 expression levels in cartilage tissue samples during OA onset, with greater levels of MMP-13 expression. Blaney et al (21) identified that the level of MMP-13 expression was significantly potentiated in cartilage tissue samples from OA model mice, indicating that MMP-13 may act as a biomarker during OA pathogenesis. Pelletier et al (23) demonstrated that MMP-13 expression levels were increased in OA model dogs. Li et al (24) demonstrated only a minimal quantity of MMP-13 in healthy cartilage tissue samples, whilst OA cartilage tissue samples exhibited potentiated MMP-13 expression levels. The current study observed significantly elevated MMP-13 expression levels in human and rat OA cartilage tissue samples, which is consistent with Blaney et al (23) and Li et al (26). Gu et al (8) demonstrated significantly reduced miR-9 expression levels in cartilage tissue samples of OA patients when compared with those of healthy tissue samples. In addition, $\mathrm{Gu}$ et al (8) performed an animal study and identified lower miR-9 expression levels in cartilage tissue samples of OA model rats. Song et al (9) observed significantly reduced miR-9 expression levels in chondrocytes from OA-derived tissues compared with those with a normal cell origin (9). The current study observed significantly reduced miR-9 expression levels in cartilage tissue samples from OA patients, which were consistent with the findings of Gu et al (8) and Song et al (9). A dual luciferase gene reporter assay demonstrated that transfection of miR-9 mimic significantly decreased relative luciferase activity in HEK 293 cells transfected with pGL3-MMP-13-3'-UTR-wt plasmid, indicating that MMP-13 is the target gene of miR-9. The injection of miR-9 agomir into the knee joint at the disease site of OA model rats significantly impaired the elevation of MMP-13 expression levels in cartilage tissue samples from the OA model, in addition to a smaller decrease in the expression level of COL2A1. These results demonstrated that application of miR-9 agomir into the knee joint effectively inhibits MMP-13 expression in the cartilage tissues of OA model rats, and reduces collagen lysis. Gu et al (8) identified that miR-9 inhibits the expression of nuclear factor $(\mathrm{NF})-\kappa \mathrm{B} 1$ by targeted binding to its 3'-UTR, further inhibiting activation of the $\mathrm{NF}-\kappa \mathrm{B}$ signaling pathway and expression levels of the downstream inflammatory factor, interleukin (IL)-6, thus inhibiting the secretion of inflammatory factor IL-6 on MMP-13. miR-9 downregulation contributes to enhancing NF- $\kappa \mathrm{B}$ signaling pathway activity, upregulating inflammatory factor and MMP-13 expression levels, and facilitating the pathogenesis of OA. Song et al (9) demonstrated that miR-9 decreased the activation of caspase- 3 and the pro-apoptotic effect on chondrocytes by protogenin (PRTG) via targeted binding to 3'-UTR, leading to expression inhibition. miR-9 downregulation is therefore involved in facilitating chondrocyte apoptosis and inducing OA pathogenesis. The current study revealed the role of miR-9 in targeted inhibition of MMP-13 expression and suppression of OA onset, which is consistent with previous studies conducted by Gu et al (8) and Song et al (9).

In conclusion, the level of miR-9 expression is suppressed whilst MMP-13 expression levels are elevated in OA cartilage tissues. miR-9 inhibits the expression level of MMP-13, thus 
suppressing its inhibitory effects on COL2A1 and enhancing COL2A1 expression levels, which consequently antagonizes the pathogenesis of OA. The results of the present study suggested that the therapeutic targeting miR-9 or MMP-13 may be beneficial for the treatment of OA. However, due to limited number of patients enrolled in the present study, large-cohort clinical studies are required to confirm these findings in the future.

\section{Acknowledgements}

This study was supported by grant no. CJN13J002.

\section{References}

1. Coudeyre E, Byers Kraus V and Rannou F: Osteoarthritis in physical medicine and rehabilitation. Ann Phys Rehabil Med 59: $133,2016$.

2. Queen RM, Sparling TL and Schmitt D: Hip, knee, and ankle osteoarthritis negatively affects mechanical energy exchange. Clin Orthop Relat Res 474: 2055-2063, 2016.

3. Wang X, Zhao X and Tang S: Inhibitory effects of EGb761 on the expression of matrix metalloproteinases (MMPs) and cartilage matrix destruction. Cell Stress Chaperones 20: 781-786, 2015.

4. Jabłońska-Trypuć A, Matejczyk M and Rosochacki S: Matrix metalloproteinases (MMPs), the main extracellular matrix (ECM) enzymes in collagen degradation, as a target for anticancer drugs. J Enzyme Inhib Med Chem 31: 177-183, 2016.

5. Li P, Deng J, Wei X, Jayasuriya CT, Zhou J, Chen Q, Zhang J, Wei L and Wei F: Blockade of hypoxia-induced CXCR4 with AMD3100 inhibits production of OA-associated catabolic mediators IL-1beta and MMP-13. Mol Med Rep 14: 1475-1482, 2016.

6. Chen YT, Hou CH, Hou SM and Liu JF: The effects of amphiregulin induced MMP-13 production in human osteoarthritis synovial fibroblast. Mediators Inflamm 2014: 759028, 2014.

7. Asahara H: Current status and strategy of microrna research for cartilage development and osteoarthritis pathogenesis. J Bone Metab 23: 121-127, 2016.

8. Gu R, Liu N, Luo S, Huang W, Zha Z and Yang J: MicroRNA-9 regulates the development of knee osteoarthritis through the NF-kappaB1 pathway in chondrocytes. Medicine (Baltimore) 95: e4315, 2016.

9. Song J, Kim D, Chun CH and Jin EJ: MicroRNA-9 regulates survival of chondroblasts and cartilage integrity by targeting protogenin. Cell Commun Signal 11: 66, 2013.

10. Peat G, Thomas E, Duncan R, Wood L, Hay E and Croft P: Clinical classification criteria for knee osteoarthritis: performance in the general population and primary care. Ann Rheum Dis 65: 1363-1367, 2006.

11. Livak KJ and Schmittgen TD: Analysis of relative gene expression data using real-time quantitative PCR and the 2(-Delta Delta C(T)) method. Methods 25: 402-408, 2001.

12. Dziri C, Aloulou I, Loubiri I, Rekik M, Zohra Ben Salah F and Abdallah A: Assessment of disability in osteoarthritis of the knee. Annals of physical and rehabilitation medicine 59s: e115, 2016.
13. Kim YH, Dorj A, Han A, Kim K and Nha KW: Improvements in spinal alignment after high tibial osteotomy in patients with medial compartment knee osteoarthritis. Gait Posture 48: 131-136, 2016.

14. Mayorga AJ, Wang S, Kelly KM and Thipphawong J: Efficacy and safety of fulranumab as monotherapy in patients with moderate to severe, chronic knee pain of primary osteoarthritis: a randomised, placebo- and active-controlled trial. Int J Clin Pract 70: 493-505, 2016.

15. van der Kraan PM, Berenbaum F, Blanco FJ, Cosimo de B, Lafeber F, Hauge E, Higginbottom A, Ioan-Facsinay A, Loughlin J, Meulenbelt I, et al: Translation of clinical problems in osteoarthritis into pathophysiological research goals. RMD Open 2: e000224, 2016.

16. Cushnaghan J and Dieppe P: Study of 500 patients with limb joint osteoarthritis. I. Analysis by age, sex, and distribution of symptomatic joint sites. Ann Rheum Dis 50: 8-13, 1991.

17. Arden N and Nevitt MC: Osteoarthritis: Epidemiology. Best Prac Res Clin Reheum 20: 3-25, 2006.

18. Montero A, Mulero JF, Tornero C, Guitart J and Serrano M: Pain, disability and health-related quality of life in osteoarthritis-joint matters: An observational, multi-specialty trans-national follow-up study. Clin Rheumatol 35: 2293-2305, 2016.

19. Frioui Mahmoudi S, Toulgui E, Ben Jeddou K, Gaddour M, Jemni S and Khachnaoui F: Quality of life for patient with knee osteoarthritis. Ann Phys Rehabil Med 59S: e158-e159, 2016.

20. Wainwright TW, Immins T and Middleton RG: A cycling and education programme for the treatment of hip osteoarthritis: A quality improvement study. Int J Orthop Trauma Nurs 23: 14-24, 2016.

21. Fu D, Zhao Y, Shen J, Cai Z and Hua Y: Comparison of venous thromboembolism after total artificial joint replacement between musculoskeletal tumors and osteoarthritis of the knee by a single surgeon. PLoS One 11: e0158215, 2016.

22. Neuhold LA, Killar L, Zhao W, Sung ML, Warner L, Kulik J, Turner J, Wu W, Billinghurst C, Meijers T, et al: Postnatal expression in hyaline cartilage of constitutively active human collagenase-3 (MMP-13) induces osteoarthritis in mice. J Clin Invest 107: 35-44, 2001.

23. Blaney Davidson EN, Remst DF, Vitters EL, van Beuningen HM, Blom AB, Goumans MJ, van den Berg WB and van der Kraan PM: Increase in ALK1/ALK5 ratio as a cause for elevated MMP-13 expression in osteoarthritis in humans and mice. J Immunol 182: 7937-7945, 2009.

24. Lim NH, Meinjohanns E, Meldal M, Bou-Gharios G and Nagase H: In vivo imaging of MMP-13 activity in the murine destabilised medial meniscus surgical model of osteoarthritis. Osteoarthritis Cartilage 22: 862-868, 2014.

25. Pelletier JP, Boileau C, Martin Boily, Brunet J, Mineau F, Geng C, Reboul P, Laufer S, Lajeunesse D and Martel-Pelletier J: The protective effect of licofelone on experimental osteoarthritis is correlated with the downregulation of gene expression and protein synthesis of several major cartilage catabolic factors: MMP-13, cathepsin K and aggrecanases. Arthritis Res Ther 7: R1091-1102, 2005.

26. Li NG, Shi ZH, Tang YP, Wang ZJ, Song SL, Qian LH, Qian DW and Duan JA: New hope for the treatment of osteoarthritis through selective inhibition of MMP-13. Curr Med Chem 18: 977-1001, 2011.

This work is licensed under a Creative Commons Attribution-NonCommercial-NoDerivatives 4.0 International (CC BY-NC-ND 4.0) License. 